http://www.jfas.info

\title{
NEUTROPHIL TO LYMPHOCYTE RATIO CORRELATE WITH SYNTAX SCORE IN PATIENTS WITH NON ST ELEVATION ACUTE CORONARY SYNDROME
}

\author{
Sh. M. Abyaneh ${ }^{1}$, M. Jalalyazdi ${ }^{*}, 2$ \\ ${ }^{1}$ Resident of Cardiology,school of Medicine, Mashhad University of Medical Sciences \\ ${ }^{2}$ MD Cardiologist, assistant professor, school of Medicine, Mashhad University of Medical
} Sciences

Published online: 15 May 2016

\begin{abstract}
Introduction :In this study we aimed to investigate the correlation between the neutrophil to lymphocyte ratio (NLR) and severity of coronary artery disease (CAD) in patients with Non ST elevation acute Coronary Syndrome (NSTE-ACS) using the SYNTAX score (SXscore).

Method: A total of 150 patients with NSTE-ACS who underwent coronary angiography were enrolled in the study. NLR was measured for all patients at presentation. The study population was then divided into 3 tertiles based on the SYNTAX trial results (1). The low syntax group $(\mathrm{n}=$ 25) was defined as those with an SXscore $\leq 22$, the intermediate syntax group $(n=78)$ was defined as an SXscore $\geq 23$ and $<33$, and the high syntax group $(n=47)$ as those with an SXscore $\geq 33$.

Result: NLR was significantly lower in patients with a low SXscore compared to patients with an intermediate SXscore or high SXscore $(2.4 \pm 2$ to $4.8 \pm 2$ and $6.9 \pm 3, \mathrm{P}<0.001)$. Linear regression analysis revealed that NLR (coefficient $\beta=0.470,95 \% \mathrm{CI}$ : $1.273-1.935, \mathrm{P}<0.001$ ) was significantly associated with the SXscore in patients with NSTE-ACS.
\end{abstract}

Author Correspondence, e-mail: author@gmail.com

doi: http://dx.doi.org/10.4314/jfas.v8i3s.283 
Conclusion: Our results indicate that NLR is independently associated with the severity of CAD in patients with NSTE-ACS.

\section{INTRODUCTION}

Non ST elevation acute coronary syndrome (NSTE-ACS) is one of the leading causes of death in patients with coronary artery disease (CAD) $(2,3)$. Patients with NSTE-ACS include patients with non ST elevation elevation myocardial infarction and patient with unstable angina. Several treatment strategies including intensive medical treatment and invasive procedures have been successful in decreasing the morbidity and mortality of NSTE-ACS (4). However, the severity of $\mathrm{CAD}$ in coronary angiography is the leading factor in determining the most useful treatment strategy. The SYNTAX score (SXscore) is an anatomic scoring system which quantifies the properties of a lesion including complexity, morphology, and location in the coronary vasculature (5). It has been shown that the SXscore may predict short and long-term mortality in patients with CAD intervention (6).

The role of inflammation in the initiation and progression of coronary atherosclerosis is well described (7-10). Increased levels of inflammatory markers have been found in association with the severity of coronary atherosclerosis and prognosis in acute coronary syndromes $(11,12)$. The neutrophil to lymphocyte ratio (NLR) is an indicator of baseline inflammatory response.

Although the predictive value of NLR on the severity of CAD in patients with STEMI and stable CAD is well known, its role in NSTE-ACS is less clear. Therefore, the aim of the present study was to investigate whether there is an association between NLR and severity of CAD in patients with NSTE-ACS using the SXscore.

\section{METHODS}

Patient selection: A total of 150 patients (100 males, mean age, $61 \pm 8$ years) were consecutively selected from among patients with NSTE-ACS who underwent coronary angiography in the catheterization laboratory of the Emam Reza Education and Research Hospital between June 2014 and July 2015. NSTE-ACS defined as any group of clinical symptoms compatible with acute myocardial ischemia and included unstable angina (UA), non-ST-segment elevation myocardial infarction (NSTEMI).To diminish any confoundersthat might influence NLR, patients with a history ofcongestive heart failure, previous percutaneous coronary 
intervention,coronary artery bypass grafting surgery history, activeinfectious disease, inflammatory or immunologic disease, cirrhosis,peripheral arterial disease, chronic obstructive pulmonarydisease, chronic kidney disease, malignancy, or cardiogenicshock on admission were excluded.

blood samples were collected at the admission to the emergency department and after fasting 12hour next day. All measurements were performed within 30 minutes after blood collection. Complete blood count, fasting blood glucose, creatinine levels, lipid profile and were analyzed for all patients.

Quantitative coronary angiography was performed using the Judkins technique by 2 experienced interventional cardiologists unaware of the clinic and laboratory results of the patients. Significant CAD was defined as $>50 \%$ stenosis in at least 1 coronary artery. Each coronary lesion producing a $\geq 50 \%$ luminal obstruction in vessels $\geq 1.5 \mathrm{~mm}$ was separately scored and added to provide the vessel SXscore. The SXscore was calculated using dedicated software that integrates the number of lesions with their specific weighting factors based on the amount of myocardium distal to the lesion according to the score of Leaman, et al and the morphologic features of each single lesion, as previously reported $(13,14)$. The study population was then divided into 3 tertiles based on the SYNTAX trial results (1). The low syntax group $(n=25)$ was defined as those with a SXscore $\leq 22$, the intermediatesyntax group $(n=78)$ as a SXscore $\geq 23$ and $<33$, and the highsyntax group $(n=47)$ as those with an SXscore $\geq 33$.

Statistical analysis was performed using the SPSS software version 15. Continuous variables are presented as the mean \pm standard deviation and categorical variables as a percentage. Linear regression analysis was performed to identify the significance of the relation between the SXscore and several variables.

\section{RESULTS}

The clinical characteristics and laboratory parameters of the patients with NSTE-ACS are listed in Table 1. NLR was significantly lower in patients with a low SXscore compared to patients with an intermediate or high SXscore $(2.4 \pm 2$ to $4.8 \pm 2$ and $6.9 \pm 3, \mathrm{P}<0.001)$. Linear regression analysis revealed that NLR (coefficient $\beta=0.470,95 \%$ CI: $1.273-1.935, \mathrm{P}<0.001$ ), age (coefficient $\beta=0.211,95 \% \mathrm{CI}: 0.110-0.235, \mathrm{P}<0.001)$ and LDL cholesterol level 
(coefficient $\beta=0.104,95 \% \mathrm{CI}: 0.004-0.052, \mathrm{P}=0.024$, Table I) were significantly associated with SXscore in patients with NSTE-ACS (Table 1).

Table 1. Clinical and laboratory parameters of the patients

\begin{tabular}{|l|l|l|l|l|}
\hline & $\begin{array}{l}\text { Mean } \pm \text { SD } \\
\text { percentage }\end{array}$ & Coefficients $\beta$ & $95 \%$ CI & P \\
\hline Age & $61 \pm 8$ years & 0.211 & $0.110-0.235$ & $<0.001$ \\
\hline Diabetes & $52(35 \%)$ & 0.036 & $-0.680-2.868$ & 0.423 \\
\hline Hypertension & $58(39 \%)$ & -0.058 & $-2.955-0.675$ & 0.324 \\
\hline Smoking & $25(17 \%)$ & 0.074 & $-0.310-3.664$ & 0.094 \\
\hline NLR & $5.3 \pm 4.7$ & 0.470 & $1.273-1.935$ & $<0.001$ \\
\hline LDL & $135 \pm 25$ & 0.104 & $0.004-0.052$ & 0.024 \\
\hline $\begin{array}{l}\text { Type of NSTE-ACS } \\
\text { unstable angina }\end{array}$ & $\begin{array}{l}23(16 \%) \\
\text { NSTEMI }\end{array}$ & 0.047 & $-0.570-3.244$ & 0.344 \\
\hline
\end{tabular}

\section{DISCUSSION}

Our results clearly demonstrate that patients with NSTE-ACS had high NLR. Our results also show that there is a significant association between NLR and severity of CAD in patients with NSTE-ACS. In this study we showed that patients with a high SXscore had higher NLR compared to those with a low or intermediate SXscore in NSTE-ACS.

Non-ST segment elevation ACS is one of the most frequent presentations of patients with CAD. Although in-hospital mortality in patients with NSTE-ACS is lower than those with ST segment elevation, 6-month mortality is similar. Moreover, 4-year mortality in patients with NSTE-ACS is two-fold higher than patients with ST segment myocardial infarction $(3,4,15)$. Therefore, risk stratification, management of patients with NSTE-ACS in the acute phase, and long-term followup are crucial to prevent increased mortality and morbidity in these patients.

The SXscore, a lesion-based angiographic scoring system, has been introduced to grade the complexity of coronaryartery disease. Since the initial trial, several trials have demonstratedthat the SXscore can be used to risk-stratify patientswith complex coronary disease. These studies have shown thatpatients with a relatively high SXscore have worse cardiovascularoutcomes, and 
that the score is an independent predictorof MACE for percutaneous coronary intervention (PCI) (16-18). Therefore, SXscore can be used in the selection of optimaltreatment by identifying those patients at highest risk of adverseevents following PCI.

NLR has been proposed as a prognostic marker and shown to be related with a pro-inflammatory state and resultant worse clinical outcomes in cardiovascular disease. NLR has been evaluated as a prognostic marker for several cardiovascular diseases including coronary artery ectasia, stable CAD, NSTEMI, and STEMI (7,19-21). NLR has also been shown in association with complexity of CAD in patients with stable CAD and acute coronary syndromes. In a recent study, Sahin, et al showed that NLR was significantly associated with severity of CAD in patients with STEMI. They also showed that NLR was an independent predictor for SXscore in patients with STEMI (16). Another study conducted by Kaya, et al showed that NLR was significantly associated with both the presence and severity of CAD in patients with stable CAD (17). In a more recent study, Altun, et al. showed that in patients with acute coronary syndrome, high sensitive troponin $\mathrm{T}$ and NLR were significantlycorrelated with the severity of CAD.

Similar to previous investigations, our study demonstrates that NLR, an indicator of systemic inflammatory response, is significantly associated with severity of CAD and may predict the SXscore in patients with NSTE-ACS. The role of inflammation in the initiation and progression of atherosclerosis is well established. During the early stages of atherosclerotic plaque development, inflammatory monocytes are provoked to move into the vascular wall by several adhesion and chemoattractant molecules released from endothelial cells (18). These monocytes differentiate to macrophages to contribute to the formation of the lipid core in advanced stages of atherosclerotic plaque development. In mature atherosclerotic plaque, various inflammatory mediators play a role in the expression of proteolytic enzymes which may weaken the fibrous cap and result in plaque rupture $(19,20)$. With this background in mind, we suggest that the inflammatory state, represented by NLR, contributes to the formation of coronary atherosclerosis in patients with NSTE-ACS.

Our study has several limitations. First, this is an observational, single-institution, cross-sectional study. second additional markers of inflammation were not assessed in our study. Also, the lack of longitudinal data regarding the association of NLR and prognosis of NSTE-ACS is another limitation of the study. 
In conclusion, NLR is a widely available parameter around the world. In patients with NSTEACS, NLR was higher in the high-SXscore group than in the low-SXscore group and was independently associated with SXscore. Also, there was a significant correlation between the SXscore and NLR ratio. Thus, we suggest that patients with NSTE-ACS who have more atherosclerotic involvement also have a higher NLR and we also suggest that a preprocedural NLR, which is an inexpensive and universally available marker, can be used for the risk stratification of patients with NSTE-ACS. Additionally, these results might play an important role in better understanding the role of inflammation in the pathogenesis of atherosclerosis and may lead to improved treatment strategies in patients with NSTE-ACS.

\section{REFERENCES}

1. Mohr FW, Morice MC, Kappetein AP, et al. Coronary artery bypassgraft surgery versus percutaneous coronary intervention inpatients with three-vessel disease and left main coronary disease:5-year follow-up of the randomised, clinical SYNTAX trial. Lancet 2013; 381: 629-38.

2. Yeh RW, Sidney S, Chandra M, Sorel M, Selby JV, Go AS. Populationtrends in the incidence and outcomes of acute myocardialinfarction. N Engl J Med 2010; 362: 2155-65.

3. Mandelzweig L, Battler A, Boyko V, et al. The second Euro HeartSurvey on acute coronary syndromes: Characteristics, treatment,and outcome of patients with ACS in Europe and the MediterraneanBasin in 2004. Eur Heart J 2006; 27: 2285-93.

4. Terkelsen CJ, Lassen JF, Nørgaard BL, et al. Mortality rates in patientswith ST-elevation vs. non-STelevation acute myocardial infarction:observations from an unselected cohort. Eur Heart J 2005;26: 18-26.

5. Kaya MG, Sahin O, Akpek M, et al. Relation between serum totalbilirubin levels and severity of coronary artery disease in patientswith non-ST-segment elevation myocardial infarction. Angiology2014; 65: 245-9.

6. van Gaal WJ, Ponnuthurai FA, Selvanayagam J, et al. The Syntaxscore predicts periprocedural myocardial necrosis during percutaneouscoronary intervention. Int J Cardiol 2009; 135: 60-5.

7. Sarli B, Baktir AO, Saglam H, et al. Neutrophil-to-lymphocyte ratiois associated with severity of coronary artery ectasia. Angiology2014; 65: 147-51. 
8. Turak O, Ozcan F, Isleyen A, et al. Usefulness of the neutrophilto-lymphocyte ratio to predict bare-metal stent restenosis. Am JCardiol 2012; 110: 1405-10.

9. Kaya MG, Akpek M, Lam YY, et al. Prognostic value of neutrophil/lymphocyte ratio in patients with ST-elevated myocardialinfarction undergoing primary coronary intervention: a prospective,multicenter study. Int J Cardiol 2013; 168: 1154-9.

10. Akpek M, Kaya MG, Lam YY, et al. Relation of neutrophil/lymphocyteratio to coronary flow to in-hospital major adverse cardiacevents in patients with ST-elevated myocardial infarction undergoingprimary coronary intervention. Am J Cardiol 2012; 110: 621-

11. Kafkas N, Demponeras C, Zoubouloglou F, Spanou L, Babalis D,Makris K. Serum levels of gelatinase associated lipocalin as indicatorof the inflammatory status in coronary artery disease. Int JInflam 2012; 2012: 189797.

12. Alfakry H, Sinisalo J, Paju S, et al. The association of serum neutrophilmarkers and acute coronary syndrome. Scand J Immunol2012; 76: 181-7.

13. Sianos G, Morel MA, Kappetein AP, et al. The SYNTAXScore:an angiographic tool grading the complexity of coronary arterydisease. EuroIntervention 2005; 1: 219-27.

14. Leaman DM, Brower RW, Meester GT, Serruys P, van den BrandM. Coronary artery atherosclerosis: severity of the disease, severityof angina pectoris and compromised left ventricular function. Circulation 1981; 63: 285-99.

15. Kurtul S, Sarli B, Baktir AO, Demirbas M, Saglam H, Doğan Y, Sahin O, Akpek M, Odabas H, Arinc H, Kaya MG. Neutrophil to lymphocyte ratio predicts SYNTAX score in patients with non-ST segment elevation myocardial infarction. Int Heart J. 2015;56(1):18-21

16. Sahin DY, Elbasan Z, Gür M, et al. Neutrophil to lymphocyte ratio is associated with the severity of coronary artery disease in patients with ST-segment elevation myocardial infarction. Angiology 2013; 64: 423-9.

17. Kaya A, Kurt M, Tanboga IH, et al. Relation of Neutrophil to Lymphocyte Ratio With the Presence and Severity of Stable Coronary Artery Disease. ClinApplThrombHemost 2013; 20: 473-7.

18. Mizuno Y, Jacob RF, Mason RP. Inflammation and the development of atherosclerosis. J AtherosclerThromb 2011; 18: 351-8. (Review) 
19. Libby P, Aikawa M. Evolution and stabilization of vulnerable atherosclerotic plaques. JpnCirc J 2001; 65: 473-9. (Review)

20. Kaya MG. Inflammation and coronary artery disease: as a new biomarker neutrophil/lymphocyte ratio. Turk Kardiyol Dern Ars 2013; 41: 191-2. (Turkish)

\section{How to cite this article:}

Abyaneh M Sh, Jalalyazdi M. Neutrophil to lymphocyte ratio correlate with syntax score in patients with non st elevation acute coronary syndrome. J. Fundam. Appl. Sci., 2016, 8(3S), 1366-1373. 